Ark. Mat., 42 (2004), 107-118

(C) 2004 by Institut Mittag-Leffler. All rights reserved

\title{
On CL-spaces and almost CL-spaces
}

\author{
Miguel Martín and Rafael Payả $\left(^{1}\right)$
}

\begin{abstract}
We find some necessary conditions for a real Banach space to be an almost CLspace. We also discuss the stability of CL-spaces and almost CL-spaces by $c_{0}$-and $l_{1}$-sums. Finally, we address the question if a space of vector-valued continuous functions can be a CL-space or an almost CL-space.
\end{abstract}

\section{Introduction}

A real or complex Banach space is said to be a $C L$-space if its unit ball is the absolutely convex hull of every maximal convex subset of the unit sphere. If the unit ball is the closed absolutely convex hull of every maximal convex subset of the unit sphere, we say that the space is an almost $C L$-space.

The concept of CL-space was introduced by R. Fullerton in 1960 [6]. Later on. $\AA$. Lima [12] introduced the almost CL-spaces, though the property specifying them had been previously used by J. Lindenstrauss [14]. Both definitions appeared only for real spaces, but they extend easily to the complex case. For general information on CL-spaces and almost CL-spaces, including the comnection with intersection properties of balls, we refer to [8], [9]: [11]. [13] and the already cited [6]. [12] and [14]. More recent results can be found in [19].

Examples of real CL-spaces are $L_{1}(\mu)$ for an arbitrary measure $\mu$, and its isometric preduals, in particular $C(K)$. where $K$ is a compact Hausdorff space (see [11, Chapter 3]). We do not know of any example of a real almost CL-space which is not a CL-space.

For complex Banach spaces, the situation is different. CL-spaces and almost CL-spaces have not received much attention in the complex case. The only result we are able to find in the literature is the characterization of finite-dimensional (real or complex) CL-spaces given by $\AA$. Lima in [12, Corollary 3.7].

(1) Research partially supported by Spanish MCYT project no. BFM12000-1467. 
In Section 2 we fix our notation and give some examples, mainly in the complex case. We show that the complex spaces $C(K)$ are always CL-spaces, whereas complex $L_{1}(\mu)$ are just almost CL-spaces. Actually; $l_{1}$ and $L_{1}[0,1]$ are not CL-spaces.

Section 3 contains some isomorphic results. We show that the dual of an infinite-dimensional real almost CL-space always contains (an isomorphic copy of) $l_{1}$. If, in addition, the dual is separable, then the space contains $c_{0}$.

In Section 4 we discuss the stability of the classes of CL-spaces and almost CL-spaces by $c_{0^{-}}$and $l_{1}$-sums. Our results are as expected, the sum is in one of the classes if and only if all the summands are. with one remarkable exception: an infinite $l_{1}$-sum of nonzero complex Banach spaces is never a CL-space.

Finally, in Section 5 we deal with the space $C(K, X)$ of continuous functions from a compact Hausdorff space $K$ into a Banach space $X$. We show that $C(K, X)$ is an almost CL-space if and only if $X$ is. The analog for CL-spaces remains open.

\section{Notation and examples}

Throughout the paper, $X$ denotes a real or complex Banach space, $B_{X}$ is its closed unit ball, $S_{X}$ its unit sphere, and $X^{*}$ the dual space. We will denote by $\mathbf{T}$ the unit sphere of the scalar field. Thus, $\mathbf{T}=\{-1.1\}$ when dealing with real spaces, while $\mathbf{T}=\{\lambda \in \mathbf{C}:|\lambda|=1\}$ in the complex case. Given a subset $A \subset X$, we write ex $A$ for the set of extreme points in $A$ and $\operatorname{co} A$ for the convex hull of $A$. Note that co $\mathbf{T} A$ is the absolutely convex hull of $A$. The closed convex hull of $A$ is denoted by $\overline{\mathrm{co}} A$. Finally, for a set $B \subset X^{*}$, we denote by $\bar{B}^{w^{*}}$ and $\overline{\mathrm{co}} w^{*} B$ the weak ${ }^{*}$-closure and the weak ${ }^{*}$-closed convex hull of $B$.

It is worth pointing out some basic facts on the definitions of CL-spaces and almost CL-spaces. By using the Hahn-Banach and Krein-Milman theorems, one can easily prove that every maximal convex subset $F$ of $S_{X}$ has the form

$$
F=\left\{x \in B_{X}: x^{*}(x)=1\right\}
$$

for some $x^{*} \in \operatorname{ex} B_{X^{*}}$. We denote by mex $B_{X^{*}}$ the set of those $x^{*} \in \operatorname{ex} B_{X^{*}}$ with the property that the set $\left\{x \in B_{X}: x^{*}(x)=1\right\}$ is a maximal convex subset of $S_{X}$. It is easy to see that mex $B_{X^{*}}$ is a boundary for $X$. that is. for every $x \in X$, there is $x^{*} \in \operatorname{mex} B_{X^{*}}$ such that $x^{*}(x)=\|x\|$. It follows that

$$
B_{X^{*}}=\overline{\operatorname{co}} w^{*} \operatorname{mex} B_{X^{*}}
$$

With the above facts in mind, $X$ is a CL-space (resp. an almost CL-space) if and only if, for every $x^{*} \in \operatorname{mex} B_{X^{*}}, B_{X}$ is the convex hull (resp. closed convex hull) of 
the set $\left\{x \in B_{X}:\left|x^{*}(x)\right|=1\right\}$. Let us also mention that maximal convex subsets of $S_{X}$ are nothing but maximal faces of $B_{X}$. This accounts for the relation of CLspaces and almost CL-spaces to the facial structure of balls. More information on this topic may be found in [1] and [11].

We shall now comment on some examples of CL-spaces and almost CL-spaces. Real CL-spaces are related to an intersection property of balls, namely the 3.2intersection property. A Banach space is said to have this property if every set of three mutually intersecting balls has nonempty intersection. The 3.2-intersection property was first investigated by O. Hanner [8] and systematically studied by J. Lindenstrauss [14] and $\AA$. Lima [11]. More references on the 3.2-intersection property are [9], [12] and [13]. In 1977. $\AA$. Lima showed that every real Banach space with the 3.2-intersection property is a CL-space [11. Corollary 3.6], but the converse is false even in the finite-dimensional case (see [8. Remark 3.6] or [19. Chapter 3]). The real space $L_{1}(\mu)$ and its isometric preduals satisfy the 3.2-intersection property. Therefore, they are CL-spaces.

To end up with the real examples, let us pose an open question. We do not know if there exists a real almost CL-space which is not a CL-space.

The situation in the complex case is quite different. Not much attention has been paid to complex CL-spaces and almost CL-spaces in the literature, so we provide the basic examples. As a matter of fact. there are complex almost CLspaces which are not CL-spaces.

Proposition 1. (i) For every compact Hausdorff space $K$, the complex space $C(K)$ is a $C L$-space.

(ii) For every finite measure $\mu$, the complex space $L_{1}(\mu)$ is an almost CL-space.

(iii) The complex spaces $l_{1}$ and $L_{1}[0,1]$ are not $C L$-spaces.

We need a lemma on the field $\mathbf{C}$ whose proof is straightforward. We write $\mathbf{D}=\{\lambda \in \mathbf{C}:|\lambda| \leq 1\}$.

Lemma 2. For every $z_{0} \in \mathbf{D}$, there exist two continuous functions $\varphi, \psi: \mathbf{D} \rightarrow \mathbf{D}$ satisfying $\left|\varphi\left(z_{0}\right)\right|=\left|\psi\left(z_{0}\right)\right|=1$ and $z=\frac{1}{2}(\varphi(z)+\psi(z))$ for every $z \in \mathbf{D}$.

Proof of Proposition 1. (i) By the well-known characterization of extreme points in $B_{C(K)^{*}}$, it suffices to show, for each $t_{0} \in K$. that $B_{C(K)}$ is the convex hull of the set $\left\{f \in B_{C(K)}:\left|f\left(t_{0}\right)\right|=1\right\}$. Indeed, given $t_{0} \in K$ and $f \in B_{C(K)}$, we take $z_{0}=f\left(t_{0}\right)$ and find functions $\varphi$ and $\psi$ as in the above lemma. Then $f=\frac{1}{2}\left(f_{1}+f_{2}\right)$. where $f_{1}=\varphi \circ f$ and $f_{2}=\psi \circ f$ satisfy $\left|f_{1}\left(t_{0}\right)\right|=\left|f_{2}\left(t_{0}\right)\right|=1$.

(ii) Let $F$ be a maximal convex subset of $S_{L_{1}(\mu)}$. Up to an isometric isomorphism, we can suppose that

$$
F=\left\{f \in L_{1}(\mu):\|f\|=1 \text { and } f \geq 0 \text { a.e. }\right\} .
$$


But the absolutely convex hull of $F$ contains a dense subset of the unit ball, namely the set of simple functions, so $B_{L_{1}(\mu)}=\overline{\mathrm{co}} \mathbf{T} F$ as desired.

(iii) It is clear that $\operatorname{ex} B_{L_{1}[0.1]^{*}}=\operatorname{mex} B_{L_{1}[0.1]^{*}}$, so the set

$$
F=\left\{f \in L_{1}[0,1]:\|f\|=1 \text { and } f \geq 0 \text { a.e. }\right\}
$$

is a maximal convex subset of $S_{L_{1}[0.1]}$. Then, it suffices to find a function $f \in$ $B_{L_{1}[0,1]}$ which cannot be obtained as an absolutely convex combination of elements in $F$. Indeed, let $f(t)=e^{2 \pi i t}$ for every $t \in[0.1]$. and suppose that we could write $f=\sum_{j=1}^{n} a_{j} \lambda_{j} f_{j}$ a.e. in $[0.1]$. where $a_{j}>0 .\left|\lambda_{j}\right|=1 . f_{j} \in F$ for $j=1,2, \ldots, n$, and $\sum_{j=1}^{n} a_{j}=1$. Then, from

$$
1=\int_{0}^{1} f(t) e^{-2 \pi i t} d t=\sum_{j=1}^{n} a_{j} \int_{0}^{1} \lambda_{j} e^{-2 \pi i t} f_{j}(t) d t \leq \sum_{j=1}^{n} a_{j} \int_{0}^{1} f_{j}(t) d t=\sum_{j=1}^{n} a_{j}=1 .
$$

we would get $\lambda_{1} e^{-2 \pi i t} f_{1}(t)=f_{1}(t)$ for almost every $t \in[0,1]$, which is clearly impossible. The case of $l_{1}$ can be treated similarly and it will become a particular case of Corollary 10.

\section{Some isomorphic results}

Our aim here is to obtain some isomorphic properties of real almost CL-spaces. We start with an easy but useful lemma.

Lemma 3. Let $X$ be an almost $C L$-space. Then $\left|x^{* *}\left(x^{*}\right)\right|=1$ for every $x^{* *} \in$ ex $B_{X^{* *}}$ and every $x^{*} \in \operatorname{mex} B_{X^{*}}$.

Proof. If $x^{*} \in \operatorname{mex} B_{X^{*}}$, we have $B_{X}=\overline{\operatorname{co}}\left\{x \in B_{X}:\left|x^{*}(x)\right|=1\right\}$ and Goldstine's theorem gives

$$
B_{X^{* *}}=\overline{\mathrm{co}}^{w^{*}}\left\{x \in B_{X}:\left|x^{*}(x)\right|=1\right\} .
$$

Now, by the 'reversed' Krein-Milman theorem we get

$$
\operatorname{ex} B_{X^{* *}} \subseteq \overline{\left\{x \in B_{X}:\left|x^{*}(x)\right|=1\right\}} u^{*} .
$$

which clearly implies $\left|x^{* *}\left(x^{*}\right)\right|=1$ for every $x^{* *} \in \operatorname{ex} B_{X} \cdots$.

Remark 4. In the real case, the converse of the above lemma holds. This follows from [12, Theorems 3.1 and 3.4].

A useful sufficient condition for a real Banach space $X$ to contain (a subspace isomorphic to) $c_{0}$ or $l_{1}$ was found in [16]. namely the existence of an infinite set $A \subset S_{X}$ where all extreme points in $B_{X}$ can only take the values \pm 1 . This fact combined with Lemma 3 yields the main result in this section. 
Theorem 5. Let $X$ be an infinite-dimensional real almost $C L$-space. Then $X^{*}$ contains $l_{1}$. If in addition $X^{*}$ is separable, then $X$ contains $c_{0}$.

Proof. Being a boundary for the infinite-dimensional space $X$. the set mex $B_{X^{*}}$ must be infinite. Then, we may apply Lemma 3 and [16. Proposition 2] to get that $X^{*}$ contains either $c_{0}$ or $l_{1}$. But a dual space contains $l_{\infty}$ (and hence $l_{1}$ ) as soon as it contains $c_{0}$ (see, e.g., [15, Proposition 2.e.8]).

To prove the second part of the proposition. observe that Lemma 3 implies that $\left\|x^{*}-y^{*}\right\|=2$ for distinct $x^{*}, y^{*} \in \operatorname{mex} B_{X^{*}}$. Therefore. if $X^{*}$ is separable. mex $B_{X^{*}}$ has to be countable. But a real infinite-dimensional space which admits a countable boundary contains $c_{0}$ by a result due to V. Fonf [5, Remark 2].

Remark 6. The above result improves those given in [16] for real Banach spaces with numerical index 1. A Banach space has numerical index 1 if every bounded linear operator $T: X \rightarrow X$ satisfies

$$
\|T\|=\sup \left\{\left|x^{*}(T x)\right|: x \in S_{X}: x^{*} \in S_{X^{*}} \text { and } x^{*}(x)=1\right\} .
$$

For more references and background we refer the reader to [2]: [3]. [4], [17] and [18]. Almost CL-spaces have numerical index 1 [17. Chapter 4]. and it is proved in [16] that the dual of an infinite-dimensional real Asplund space with numerical index 1 contains $l_{1}$.

The behaviour of CL-spaces or almost CL-spaces under duality is unclear to us. It was shown by $\AA$. Lima [12, Corollary 3.6] that a real Banach space $X$ is an almost CL-space provided that $X^{*}$ is a CL-space. We are not aware of any relevant result in the opposite direction. Next we get such a result, under an additional assumption.

Proposition 7. Let $X$ be a Banach space not containing $l_{1}$. If $X$ is an almost CL-space, then $X^{*}$ is an almost $C L$-space.

Proof. For $x^{* *} \in \operatorname{mex} B_{X^{* *}}$, we need to show that $B_{X^{*}}=\overline{\mathrm{co}} H$. where

$$
H=\left\{x^{*} \in B_{X^{*}}:\left|x^{* *}\left(x^{*}\right)\right|=1\right\} .
$$

We have mex $B_{X^{*}} \subseteq H$ by Lemma 3. Moreover, since mex $B_{X^{*}}$ is a boundary for a space $X$ not containing $l_{1}$, we can use [7. Theorem III.1] to get that $B_{X^{*}}=$ $\overline{\mathrm{co}} \operatorname{mex} B_{X^{*}}$, and we are done.

\section{Sums of CL-spaces and almost CL-spaces}

This section is devoted to study the stability of the classes of CL-spaces and almost CL-spaces by $c_{0^{-}}$and $l_{1}$-sums. Given an arbitrary family $\left\{X_{\lambda}: \lambda \in \Lambda\right\}$ of 
Banach spaces, we denote by $\left[\bigoplus_{\lambda \in \Lambda} X_{\lambda}\right]_{c_{0}}$ (resp. $\left[\bigoplus_{\lambda \in \Lambda} X_{\lambda}\right]_{l_{1}}$ ) the $c_{0}$-sum (resp. $l_{1}$-sum) of the family. In case $\Lambda$ has just two elements, we use the simpler notation $X \oplus_{\infty} Y$ or $X \oplus_{1} Y$.

With respect to $c_{0}$-sums, we have the following result.

Proposition 8. Let $\left\{X_{\lambda}: \lambda \in \Lambda\right\}$ be a family of Banach spaces and let $X=$ $\left[\bigoplus_{\lambda \in \Lambda} X_{\lambda}\right]_{c_{0}}$. Then, $X$ is a CL-space (resp. an almost CL-space) if and only if $X_{\lambda}$ is a CL-space (resp. an almost CL-space) for every $\lambda \in \Lambda$.

Proof. For each $\lambda \in \Lambda$, we write $I_{\lambda}$ for the injection of $X_{\lambda}$ into $X$, and $P_{\lambda}$ will be the projection of $X$ onto $X_{\lambda}$. Under the natural identification $X^{*}=\left[\oplus_{\lambda \in \Lambda} X_{\lambda}^{*}\right]_{l_{1}}$, $P_{\lambda}^{*}$ becomes the injection of $X_{\lambda}^{*}$ into $X^{*}$. Using the well-known fact that

$$
\operatorname{ex} B_{X^{*}}=\bigcup_{\lambda \in \Lambda} P_{\lambda}^{*}\left(\operatorname{ex} B_{X_{\lambda}^{*}}\right)
$$

one can easily check that a convex subset $F$ of $S_{X}$ is maximal if and only if there exist $\lambda \in \Lambda$ and a maximal convex subset $F_{\lambda}$ of $S_{X_{\lambda}}$ such that

$$
F_{\lambda}=P_{\lambda}(F) \quad \text { and } \quad F=P_{\lambda}^{-1}\left(F_{\lambda}\right) \cap B_{X} .
$$

Suppose first that every $X_{\lambda}$ is an almost CL-space, and fix a maximal convex subset $F$ of $S_{X}$. Then, we can find $\lambda \in \Lambda$ and a maximal convex subset $F_{\lambda}$ of $S_{X_{\lambda}}$ satisfying (1), so $B_{X_{\lambda}}=\overline{\mathrm{co}} \mathbf{T} F_{\lambda}$. Now, given $x \in B_{X}$ and $\varepsilon>0$, take $y_{\lambda} \in \operatorname{co} \mathbf{T} F_{\lambda}$ such that $\left\|y_{\lambda}-P_{\lambda}(x)\right\|<\varepsilon$, and consider $y=x-I_{\lambda}\left(P_{\lambda}(x)\right)+I_{\lambda}\left(y_{\lambda}\right)$. It is clear that $y \in B_{X}$ and $P_{\lambda}(y)=y_{\lambda}$, so $y \in \operatorname{co} \mathbf{T} F$. Moreover, $\|y-x\|<\varepsilon$, so we have shown that $B_{X}=\overline{\mathrm{CO}} \mathbf{T} F$ and $X$ is an almost CL-space. If every $X_{\lambda}$ is a CL-space, then we can repeat the above argument with $\varepsilon=0$ to get that $X$ is a CL-space.

Conversely, suppose that $X$ is an almost CL-space. Fix $\lambda \in \Lambda$ and let $F_{\lambda}$ be a maximal convex subset of $S_{X_{\lambda}}$. Then, the set $F$ given by (1) is a maximal convex subset of $S_{X}$, so $B_{X}=\overline{\mathrm{co}} \mathbf{T} F$. Now, given $x_{\lambda} \in B_{X_{\lambda}}$ and $\Xi>0$, there exists $y \in \operatorname{co} \mathbf{T} F$ such that $\left\|y-I_{\lambda}\left(x_{\lambda}\right)\right\|<\varepsilon$. Then

$$
P_{\lambda}(y) \in \operatorname{co} \mathbf{T} F_{\lambda} \text { and }\left\|P_{\lambda}(y)-x_{\lambda}\right\|<\varepsilon,
$$

so $B_{X_{\lambda}}=\overline{\operatorname{co}} \mathbf{T} F_{\lambda}$ and $X_{\lambda}$ is an almost CL-space. The same argument with $\varepsilon=0$ gives that $X_{\lambda}$ is a CL-space if $X$ is.

The examples in Proposition 1 tell us that the situation for $l_{1}$-sums cannot be so tidy. Actually, we have the following result. 
Proposition 9. Let $\left\{X_{\lambda}: \lambda \in \Lambda\right\}$ be a family of nonzero Banach spaces and let $X=\left[\bigoplus_{\lambda \in \Lambda} X_{\lambda}\right]_{l_{1}}$. Then the following statements hold:

(i) The space $X$ is an almost CL-space if and only if every $X_{\lambda}$ is.

(ii) In the real case, $X$ is a CL-space if and only if every $X_{\lambda}$ is.

(iii) In the complex case, $X$ is a CL-space if and only if every $X_{\lambda}$ is and the set $\Lambda$ is finite.

Proof. Once again we write $I_{\lambda}$ and $P_{\lambda}$ for the natural injections and projections. Now, $X^{*}=\left[\oplus_{\lambda \in \Lambda} X_{\lambda}^{*}\right]_{l_{\infty}}, I_{\lambda}^{*}$ is the natural projection of $X^{*}$ onto $X_{\lambda}^{*}$, and the relationship between extreme points in $B_{X^{*}}$ and $B_{X_{\lambda}^{*}}$ is the following:

$$
x^{*} \in \operatorname{ex} B_{X^{*}} \Longleftrightarrow I_{\lambda}^{*}\left(x^{*}\right) \in \operatorname{ex} B_{X_{\lambda}^{*}} \text { for all } \lambda \in \Lambda \text {. }
$$

With this in mind, it is easy to check that maximal convex subsets of $S_{X}$ are exactly the sets of the form

$$
F=\left\{x \in S_{X}: P_{\lambda}(x) \in\left\|P_{\lambda}(x)\right\| F_{\lambda} \text { for all } \lambda \in \Lambda\right\},
$$

where $F_{\lambda}$ is a maximal convex subset of $S_{X_{\lambda}}$ for all $\lambda \in \Lambda$.

(i) Suppose first that $X$ is an almost CL-space and, for fixed $\mu \in \Lambda$. let $F_{\mu}$ be a. maximal convex subset of $S_{X_{\mu}}$. For every $\lambda \neq \mu$ we choose an arbitrary maximal convex subset $F_{\lambda}$ of $S_{X_{\lambda}}$ and consider the maximal convex subset $F$ of $S_{X}$ given by (2). Note that

$$
P_{\mu}(x) \in\left\|P_{\mu}(x)\right\| F_{\mu} \subset \operatorname{co} \mathbf{T} F_{\mu}
$$

for all $x \in F$, so

$$
P_{\mu}(\operatorname{co} \mathbf{T} F) \subseteq \operatorname{co} \mathbf{T} F_{\mu} .
$$

Now, using that $B_{X}=\overline{\mathrm{co}} \mathbf{T} F$, we have

$$
B_{X_{\mu}}=P_{\mu}\left(B_{X}\right)=P_{\mu}(\overline{\operatorname{co}} \mathbf{T} F) \subseteq \overline{P_{\mu}(\operatorname{co} \mathbf{T} F)} \subseteq \overline{\operatorname{co}} \mathbf{T} F_{\mu}
$$

and we have shown that $X_{\mu}$ is an almost CL-space. Note that if $X$ is a CL-space, then

$$
B_{X_{\mu}}=P_{\mu}(\cot F) \subseteq \cot F_{\mu}
$$

and $X_{\mu}$ is a CL-space.

Conversely, suppose that every $X_{\lambda}$ is an almost CL-space, and let $F$ be a maximal convex subset of $S_{X}$. Then $F$ has the form given in (2) where $F_{\lambda}$ is a maximal convex subset of $S_{X_{\lambda}}$ for each $\lambda \in \Lambda$. Since $B_{X_{\lambda}}=\overline{\operatorname{co}} \mathbf{T} F_{\lambda}$ and $I_{\lambda}\left(F_{\lambda}\right) \subseteq F$ for every $\lambda \in \Lambda$, we have

$$
\bigcup_{\lambda \in \Lambda} I_{\lambda}\left(B_{X_{\lambda}}\right) \subseteq \bigcup_{\lambda \in \Lambda} \overline{\operatorname{co}} \mathbf{T} I_{\lambda}\left(F_{\lambda}\right) \subseteq \overline{\operatorname{co}} \mathbf{T} F
$$


Now, just recall that $B_{X}$ is the closed convex hull of $\bigcup_{\lambda \in \Lambda} I_{\lambda}\left(B_{X_{\lambda}}\right)$, so $B_{X} \subseteq$ $\overline{\mathrm{CO}} \mathbf{T} F$ and $X$ is an almost CL-space. Observe that, if $\Lambda$ is finite, then $B_{X}=$ $\operatorname{co\bigcup } \bigcup_{\lambda \in \Lambda} I_{\lambda}\left(B_{X_{\lambda}}\right)$ and the above argument allows us to obtain $B_{X}=\operatorname{co} \mathbf{T} F$ from $B_{X_{\lambda}}=\operatorname{co} \mathbf{T} F_{\lambda}$ for all $\lambda$. Therefore, finite $l_{1}$-sums of CL-spaces are CL-spaces.

(ii) One implication has already been proved. For the converse, suppose that each $X_{\lambda}$ is a real CL-space, let $F$ be a maximal convex subset of $S_{X}$ and write $F$ as in (2). Fix $x \in S_{X}$ and use that $B_{X_{\lambda}}=\operatorname{co}\left(F_{\lambda} \cup-F_{\lambda}\right)$ to write

$$
P_{\lambda}(x)=\left\|P_{\lambda}(x)\right\|\left(t_{\lambda} y_{\lambda}-\left(1-t_{\lambda}\right) \hat{\sim}_{\lambda}\right) . \quad \lambda \in \Lambda .
$$

where $y_{\lambda}, z_{\lambda} \in F_{\lambda}$ and $0 \leq t_{\lambda} \leq 1$ for all $\lambda$. It follows that

$$
x=\sum_{\lambda \in \Lambda} I_{\lambda}\left(P_{\lambda}(x)\right)=y-z .
$$

where

$$
y=\sum_{\lambda \in \Lambda}\left\|P_{\lambda}(x)\right\| t_{\lambda} I_{\lambda}\left(y_{\lambda}\right) \quad \text { and } \quad z=\sum_{\lambda \in \Lambda}\left\|P_{\lambda}(x)\right\|\left(1-t_{\lambda}\right) I_{\lambda}\left(z_{\lambda}\right)
$$

satisfy that

$$
\|y\|+\|z\|=\sum_{\lambda \in \lambda}\left\|P_{\lambda}(x)\right\|=1 .
$$

For each $\lambda$ we have $P_{\lambda}(y)=\left\|P_{\lambda}(y)\right\| y_{\lambda} \in\left\|P_{\lambda}(y)\right\| F_{\lambda}$ and it clearly follows that we may write $y=\|y\| y_{0}$ with $y_{0} \in F$. Similarly; $z=\|z\| z_{0}$ with $z_{0} \in F$. and we get

$$
x=\|y\| y_{0}-\|z\| z_{0} \in \operatorname{co}(F \cup-F) .
$$

We have shown that $S_{X} \subset \operatorname{co}(F \cup-F)$, so $B_{X}=\operatorname{co}(F \cup-F)$ and $X$ is a CL-space.

(iii) We have already seen that every $X_{\lambda}$ is a CL-space if $X$ is, and that the converse holds for finite $\Lambda$. Thus, it only remains to show that $X$ cannot be a CL-space if $\Lambda$ is infinite. Without loss of generality we just consider the case $\Lambda=\mathbf{N}$. Indeed, choose an infinite countable subset $\Lambda_{0}$ of $\Lambda$ and write $X=Y \bigodot_{1} Z$ with $Y=\left[\oplus_{\lambda \in \Lambda_{0}} X_{\lambda}\right]_{i_{1}}$ and suitable $Z$. Were $X$ a CL-space. our above results show that $Y$ would be a CL-space as well. Thus. suppose that $X=\left[\bigoplus_{k \in \mathbf{N}} X_{k}\right]_{l_{1}}$ is a CL-space to get a contradiction.

For each $k \in \mathbf{N}$, we fix $e_{k}^{*} \in \operatorname{mex} B_{X_{k}^{*}}$ and build up the set

$$
F=\left\{x \in S_{X}: e_{k}^{*}\left(P_{k}(x)\right)=\left\|P_{k}(x)\right\| \text { for all } k \in \mathbf{N}\right\},
$$

a maximal convex subset of $S_{X}$. Now. let

$$
x=\sum_{k=1}^{\infty} \frac{\alpha_{k}}{2^{k}} I_{k}\left(e_{k}\right)
$$


where $\left(\alpha_{k}\right)_{k=1}^{\infty}$ is a sequence of distinct elements in $\mathbf{T}$ : $\epsilon_{h} \in S_{X_{k}}$ and $e_{k}^{*}\left(e_{k}\right)=1$ for every $k \in \mathbf{N}$. Since $B_{X}=\operatorname{co} \mathbf{T} F$, we can write $x$ in the form

$$
x=\sum_{j=1}^{m} \beta_{j} x^{(j)}
$$

where

$$
\beta_{1}, \beta_{2}, \ldots, \beta_{m} \in \mathbf{C}, \quad \sum_{j=1}^{m}\left|\beta_{j}\right|=1 \quad \text { and } \quad x^{(1)} . x^{(2)} \ldots . x^{(m)} \in F .
$$

We deduce that, for every $k \in \mathbf{N}$,

$$
\frac{\alpha_{k}}{2^{k}}=e_{k}^{*}\left(P_{k}(x)\right)=\sum_{j=1}^{m} \beta_{j} e_{k}^{*}\left(P_{k}\left(x^{(j)}\right)\right)=\sum_{j=1}^{m} \beta_{j}\left\|P_{k}\left(x^{(j)}\right)\right\| .
$$

so

$$
1=\sum_{k=1}^{\infty} \frac{1}{2^{k}}=\sum_{j=1}^{m} \sum_{k=1}^{\infty} \overline{\alpha_{k}} \beta_{j}\left\|P_{k}\left(x^{(j)}\right)\right\| \leq \sum_{j=1}^{m} \sum_{k=1}^{\infty}\left|3_{j}\right|\left\|P_{k}\left(x^{(j)}\right)\right\|=\sum_{j=1}^{m}\left|\beta_{j}\right|\left\|x^{(j)}\right\|=1
$$

It follows that

$$
\overline{\alpha_{k}} \beta_{j}\left\|P_{k}\left(x^{(j)}\right)\right\|=\left|\beta_{j}\right|\left\|P_{k}\left(x^{(j)}\right)\right\|
$$

for every $j \in\{1,2, \ldots, m\}$ and every $k \in \mathbf{N}$. Since

$$
0 \neq P_{k}(x)=\sum_{j=1}^{m} 3_{j} P_{k}\left(x^{(j)}\right)
$$

for each $k \in \mathbf{N}$, there must exist some $j \in\{1,2 \ldots . m\}$ such that $\beta_{j} P_{k}\left(x^{(j)}\right) \neq 0$, and (3) gives $\alpha_{k}=\beta_{j} /\left|\beta_{j}\right|$. This contradicts the choice of the sequence $\left(\alpha_{k}\right)_{k=1}^{x}$.

Let us point out the following consequence.

Corollary 10. Let $\mu$ be a measure. The complex space $L_{1}(\mu)$ is a CL-space if and only if $\operatorname{dim} L_{1}(\mu)<\infty$.

Proof. By [10, pp. 136], we have

$$
L_{\mathbf{1}}(\mu) \equiv l_{1}(\Gamma) \ominus_{1}\left[\bigoplus_{\alpha \in A} L_{1}\left([0,1]^{m_{a}}\right)\right]_{l_{1}}
$$


for some index set $\Gamma$ and some set $A$ of cardinal numbers $m_{\alpha} \geq \aleph_{0}$. If $L_{1}(\mu)$ is a CLspace, Proposition 9 gives us that $\Gamma$ and $A$ are finite and $L_{1}\left([0.1]^{m_{\alpha}}\right)$ is a CL-space for every $\alpha \in A$. Now, since Theorem 14.10 of [10] gives

$$
L_{1}\left([0,1]^{m_{a}}\right) \equiv\left[\bigoplus_{k=1}^{\infty} L_{1}\left([0,1]^{m_{k}}\right)\right]_{l_{1}},
$$

where $m_{k}=m_{\alpha}$ for every $k \in \mathbf{N}$, another application of Proposition 9 tells us that $L_{1}\left([0,1]^{m_{\alpha}}\right)$ is not a CL-space. Therefore. $A=\emptyset, L_{1}(\mu) \equiv l_{1}(\Gamma)$ for a suitable finite set $\Gamma$, and $\operatorname{dim} L_{1}(\mu)<\infty$. The converse result is clear.

\section{The space $C(K, X)$}

Given a compact Hausdorff space $K$ and a Banach space $X$, we consider the Banach space $C(K, X)$ of all continuous functions from $K$ into $X$, endowed with its natural supremum norm.

Proposition 11. Let $K$ be a compact Hausdorff space and let $X$ be a Banach space. Then the following statements hold:

(i) The space $C(K, X)$ is an almost $C L$-space if and only if $X$ is.

(ii) If $C(K, X)$ is a CL-space, then $X$ is also a CL-space.

Proof. Let us write $Y=C(K, X)$ and recall that the extreme points in $B_{Y^{*}}$ are functionals of the form $f \mapsto x^{*}(f(t))$, where $x^{*} \in \operatorname{ex} B_{X^{*}}$ and $t \in K$ (see [20, Theorem 1.1]). With this in mind, it is easy to check that the maximal convex subsets of $S_{Y}$ are just the sets of the form

$$
\mathcal{F}=\left\{f \in S_{Y}: f(t) \in F\right\}
$$

where $t \in K$ and $F$ is a maximal convex subset of $S_{X}$.

Suppose first that $X$ is an almost CL-space. Fix a maximal convex subset $\mathcal{F}$ of $S_{Y}$ and let $t$ and $F$ be as in (4). Since $B_{X}=\overline{\mathrm{co}} \mathbf{T} F$, given $f \in S_{Y}$ and $\varepsilon>0$ we can find $x \in \operatorname{co} \mathbf{T} F$ such that

$$
\|x-f(t)\|<\varepsilon
$$

and build a continuous function $\varphi: K \rightarrow[0,1]$ such that

$$
\varphi(t)=1 \quad \text { and } \quad \varphi(s)=0 \text {, if }\|x-f(s)\| \geq \varepsilon \text {. }
$$

If we define $g \in S_{Y}$ by

$$
g(s)=\varphi(s) x+(1-\varphi(s)) f(s) . \quad s \in K
$$


it is easy to see that $g \in \operatorname{co} \mathbf{T} \mathcal{F}$ and $\|f-g\| \leq \Xi$. Therefore. $B_{Y^{\prime}}=\overline{\mathrm{co}} \mathbf{T} \mathcal{F}$ and $Y$ is an almost CL-space.

Now, suppose that $Y$ is an almost CL-space. Fix a maximal convex subset $F$ of $S_{X}$, and consider the maximal convex subset $\mathcal{F}$ of $S_{Y}$ given by (4) for an arbitrary $t \in K$. Further, fix $x \in B_{X}$ and $\varepsilon>0$, consider $f=x \chi_{K} \in B_{Y}$ and use that $B_{Y}=\overline{\mathrm{co}} \mathbf{T} \mathcal{F}$ to find $g \in \operatorname{co} \mathbf{T} \mathcal{F}$ such that $\|f-g\|<\varepsilon$. Then $g(t) \in \operatorname{co} \mathbf{T} F$ and $\|g(t)-x\|<\varepsilon$, so $B_{X}=$ $\overline{\mathrm{co}} \mathbf{T} F$ and $X$ is an almost CL-space.

In the case when $Y$ is a CL-space, we can repeat the above argument with $\varepsilon=0$ to prove that $X$ is also a CL-space.

To finish the paper, let us mention that we do not know whether $C(K, X)$ is a CL-space whenever $X$ is.

Acknowledgement. The authors wish to express their gratitude to the referee for pointing out Corollary 10.

\section{References}

1. Alfsen, E. and Effros, E., Structure in real Banach spaces, Ann. of Math. 96 (1972), 98-173.

2. Bonsall, F. F. and Duncax, J., Numerical Ranges of Operators on Normed Spaces and of Elements of Normed Algebras, London Math. Soc. Lecture Note Ser.

2, Cambridge Univ. Press, London-New York. 1971.

3. Bonsall, F. F. and DUNCAN, J., Numerical Ranges II. London Math. Soc. Lecture Note Ser. 10, Cambridge Univ. Press, London-New York, 1973.

4. Duncan, J., MCGregor, C. M., Pryce, J. D. and White. A. J., The numerical index of a normed space, J. London Math. Soc. 2 (1970), 481-488.

5. Fonf, V. P., Weakly extremal properties of Banach spaces, Mat. Zametki 45:6 (1989), 83-92, 112 (Russian). English transl.: Math. Notes 45 (1989), 488-494.

6. Fullerton, R. E., Geometrical characterizations of certain function spaces, in Proc. Internat. Sympos. Linear spaces (Jerusalem, 1960), pp. 227-236, Jerusalem Academic Press, Jerusalem; Pergamon Press, Oxford, 1961.

7. Godefroy, G., Boundaries of a convex set and interpolation sets, Math. Ann. 277 (1987), 173-184.

8. Hanner, O., Intersections of translates of convex bodies. Math. Scand. 4 (1956). $65-87$.

9. Hansen, A. B. and Lina, A., The structure of finite dimensional Banach spaces with the 3.2. intersection property: Acta Math. 146 (1981), 1-23.

10. LACEX, H. E., The Isometric Theory of Classical Banach Spaces, Springer-Verlag, Berlin-Heidelberg-New York. 1974.

11. LIMA, $\AA$., Intersection properties of balls and subspaces in Banach spaces, Trans. Amer. Math. Soc. 227 (1977). 1-62.

12. LimA, A., Intersection properties of balls in spaces of compact operators, Ann. Inst. Fourier (Grenoble) 28:3 (1978), 35-65. 
13. LImA, $\AA$., On extreme operators on finite-dimensional Banach spaces whose unit balls are polytopes, Ark. Mat. 19 (1981), 97-116.

14. Lindenstrauss, J., Extension of Compact Operators, Mem. Amer. Math. Soc. 48, Amer. Math. Soc., Providence. R. I., 1964.

15. Lindenstrauss, J. and Tzafriri, L.. Classical Banach Spaces I: Sequence Spaces, Springer-Verlag, Berlin-New York, 1977.

16. López, G., Martín, M. and Payá. R.. Real Banach spaces with numerical index 1, Bull. London Math. Soc. 31 (1999). 207-212.

17. Martín, M., A survey on the numerical index of a Banach space, in III Congress on Banach Spaces (Jarandilla de la Vera, 1998). Extracta Math. 15, pp. 265276, Universidad de Extremadura, Departamento de Matemáticas, Badajoz, 2000.

18. Martín, M. and PAYÁ, R., Numerical index of vector-valued function spaces, Studia Math. 142 (2000): 269-280.

19. ReIsner, S., Certain Banach spaces associated with graphs and CL-spaces with 1unconditional bases, J. London Math. Soc. 43 (1991). 137-148.

20. Ruess, W. M. and Stegall, C. P., Extreme points in duals of operator spaces, Math. Ann. 261 (1982), 535-546.

Received November 15, 2002

in revised form February 26, 2003
Miguel Martín

Departamento de Análisis Matemático

Facultad de Ciencias

Universidad de Granada

ES-18071 Granada

Spain

email: mmartins âugr.es

Rafael Payá

Departamento de Análisis Matemático

Facultad de Ciencias

Universidad de Granada

ES-18071 Granada

Spain

email: rpaya ậugr.es 